\title{
Preoperative coping strategies and distress predict postoperative pain and morphine consumption in women undergoing abdominal gynecologic surgery
}

\author{
Lorenzo Cohen ${ }^{\mathrm{a}, *}$, Rachel T. Fouladi ${ }^{\mathrm{b}}$, Joel Katz ${ }^{\mathrm{c}, \mathrm{d}, \mathrm{e}, \mathrm{f}}$ \\ ${ }^{2}$ Department of Behavioral Science The University of Texas, M.D. Anderson Cancer Center, Box 243, 1515 Holcombe Boulevard, Houston, TX 77030, USA \\ ${ }^{b}$ Department of Psychologv, Simon Fraser University, Vancouver, BC, Canada \\ ${ }^{\mathrm{c}}$ Department of Psychology and School of Kinesiology and Health Science, York University, Toronto, ON, Canada \\ ${ }^{\mathrm{d} D e p a r t m e n t}$ of Anesthesia and Pain Management, Toronto General Hospital, Toronto, ON, Canada \\ ${ }^{2}$ Mount Sinai Hospital, Toronto, ON, Canada \\ ${ }^{\mathrm{f}}$ Department of Anesthesia, University of Toronto, Toronto, ON, Canada
}

\begin{abstract}
Objectives: The aim of the present study was to predict postoperative pain and morphine consumption based on preoperative psychosocial factors. Methods: One hundred and twentytwo women completed measures of distress and coping 1 week before major abdominal gynecological surgery by laparotomy. Forty-eight hours after surgery, measures of pain and negative affect (NA) were completed, and morphine consumption was recorded from a patient-controlled analgesia pump. Four weeks after surgery, measures of pain and NA were completed. Results: Multivariate analyses revealed that preoperative self-distraction coping ( $P=.039$ ) positively predicted postoperative pain levels in the hospital, after accounting for the effects of age, concurrent
\end{abstract}

NA, and morphine consumption. Emotional support $(P=.031)$ and religious-based coping $(P=.036)$ positively predicted morphine consumption in the hospital, after accounting for the effects of age, concurrent NA, and pain levels. Preoperative distress $(P<.04$ to .008$)$ and behavioral disengagement $(P=.034)$, emotional support $(P=.049)$, and religious-based coping $(P=.001)$ positively predicted pain levels 4 weeks after surgery, after accounting for the effects of age and concurrent NA. Conclusion: The results suggest that preoperative psychosocial factors are associated with postoperative pain and morphine consumption.

\section{Introduction}

Until recently, the role of presurgical psychosocial factors in predicting adjustment to the acute outcomes after surgery has been limited to the investigation of anxiety and related states. The results of these studies are equivocal. For example, in some studies, state-based factors, such as anxiety and anticipatory stress, have been found to be positively associated with physical complaints [1] and

\footnotetext{
* Corresponding author. Tel.: +1 713745 4260; fax: +1 7137454286 .

E-mail address: Icohen(@)mdanderson.org (L. Cohen).
}

postoperative pain and/or analgesic consumption [2-8]. In other studies, however, no association has been found between preoperative anxiety and pain or analgesic consumption $[3,9-15]$.

Part of the difficulty in accurately determining the nature of the relationship between preoperative anxiety and postoperative pain arises because studies have failed to assess other important variables in the postoperative period that are known to influence pain and analgesic consumption. For example, the preoperative anxiety that may be associated with postoperative pain could reflect a dispositional characteristic of the individual that is associated with a 
generalized increased reactive state to stressful situations, which may include increased pain levels. Alternatively, postoperative anxiety may lead to increased pain reporting, independent of the dispositional anxiety level.

There has been considerably less research examining presurgical coping behaviors as predictors of postoperative adjustment. It has been suggested that avoidant coping (e.g., not thinking about the upcoming surgery) is associated with less preoperative anxiety and better short-term recovery than is vigilant-style coping $[16,17]$. However, in most studies, the effects of coping style have not been disentangled from their association with anxiety in predicting postoperative recovery measures.

The main aim of the present study was to determine whether presurgical psychosocial factors were associated with postoperative pain reports and analgesic consumption after adjusting for the effects of other medical factors and concurrent variables. We hypothesized that presurgical measures of distress and general coping behaviors would be associated with postoperative pain and analgesic consumption, after adjusting for the concurrent factors. We were particularly interested in determining the extent to which the endorsement of specific coping strategies would predict postoperative pain and analgesic consumption after controlling for the effects of the distress measures before surgery and negative affect (NA) at the time pain was assessed. Factors that are amenable to change were of particular interest because knowledge of modifiable psychological and behavioral characteristics associated with adjustment could aid in the design of more effective, targeted preoperative intervention programs.

\section{Method}

\section{Participants}

The present study was conducted in the context of a randomized, double-blind, placebo-controlled trial examining the benefits of preincisional epidural anesthesia for women undergoing major abdominal surgery. Patients scheduled for major gynecological surgical procedures by laparotomy were eligible for recruitment into the study. Inclusion criteria were ASA physical status I-II, age between 19 and 75 years, weight between 45 and $90 \mathrm{~kg}$, height between 150 and $175 \mathrm{~cm}$, body mass index less than or equal to 30, and ability to speak and read English. Exclusion criteria were contraindications to patient-controlled analgesic (PCA) morphine, or regional anesthesia, history of a major psychiatric disorder, history of a substance use disorder, and current opioid use.

The same general anesthesia protocol was used in all patients, who were randomly assigned to either receive (1) preincisional epidural lidocaine with epinephrine and fentanyl, with epidural saline given 35-40 min after incision; (2) preincisional epidural injections of normal saline, with epidural lidocaine with epinephrine and fentanyl given 35-40 min after the incision; or (3) sham epidural catheter and administration of saline before and after surgery. Importantly, when asked to guess the group to which they had been assigned, patients performed at a chance level. The study was approved by The Toronto Hospital Committee for Research on Human Subjects.

\section{Procedure}

\section{Preoperative assessments}

A member of the research team approached prospective patients at their preadmission appointment approximately 7-10 days before surgery. Following informed written consent, patients completed the preassessment battery of questionnaires, which included measures of coping and distress (general mental health, intrusive and avoidant thoughts about surgery, and state-based NA).

\section{Postoperative assessments}

Pain and NA were assessed by administering self-report measures $48 \mathrm{~h}$ and 4 weeks after surgery. Patients were contacted by telephone for the 4-week follow-up assessment, with a maximum of five attempts to contact each patient. Analgesic consumption was assessed up to $48 \mathrm{~h}$ after surgery.

\section{Measures}

\section{Presurgical psychosocial measures}

Coping tendency or style was measured with the BriefCOPE. The COPE, which has been validated in women with breast cancer, measures a set of conceptually distinct coping subscales that include active coping, use of social support, positive reframing, planning, acceptance, venting, humor, religious-based coping, and avoidant coping (selfdistraction, denial, substance use, and behavioral disengagement; [18.19]). The internal reliability of most items was adequate, with the lowest reliability for acceptance (.49), venting (.57), and positive reframing (.63). Reliability for the other scales ranged from .67 to .90 . Participants completed the items in reference to how they have been coping with the stress of their upcoming surgery.

The Mental Health Inventory (MHI; [20]) is an 18-item scale that measures symptoms of psychological distress and well-being along five dimensions - anxiety, depression, loss of behavioral/emotional control, positive affect, and interpersonal ties-and also yields a total score [21]. Participants responded on the basis of how often in the past month they had experienced each symptom. The total score, which was used in this study, ranges from 0 to 108, with higher scores indicative of better mental health. The MHI is extremely reliable (Cronbach's $\alpha=.96$ ).

The Impact of Events Scale (IES) is a 15-item, self-report scale that assesses two categories of cognitive responses to stressful events: intrusion (intrusively experienced ideas, images, feelings, or bad dreams) and avoidance (consciously 
recognized avoidance of certain ideas, feelings, or situations; [22]). Patients in the present study were asked to rate the frequency of intrusive thoughts and avoidance behaviors in relation to their upcoming surgery, with higher scores representing worse functioning. The correlation between the Intrusion and Avoidance subscales was .77, hence, the IES total score, which is simply the sum of the two subscale scores, was used for the analyses.

NA was assessed using a 26-item stress scale that has been shown to be a reliable measure of acute distress $[23,24]$. Each item was rated on a five-point scale ranging from not at all to extremely. Participants rated the level of stress-related feelings that they were currently experiencing along affective and somatic dimensions. Higher scores are indicative of increased NA. The NA questionnaire had good internal consistency at each time point (Cronbach's $\alpha$ : preoperative $=.95 ; 48 \mathrm{~h}=.92$; and 4-week follow-up assessment $=.93$ ). The NA questionnaire was completed at baseline, $48 \mathrm{~h}$, and 4 weeks after surgery.

\section{Measures of pain and morphine consumption}

The McGill Pain Questionnaire (MPQ) was developed by Melzack $[25,26]$ to quantitatively and qualitatively measure the experience of pain. The MPQ yields two global scores, the pain rating index (PRI-T) and the present pain intensity (PPI), which are valid and reliable measures of pain [25]. The PRI-T is the sum of the rank values of the words chosen from 20 sets of qualitative words, with each set containing two to six adjectives that describe the sensory, affective, and valuative properties of pain. For the present analysis, we report data from the PRI-T.

Patients were assessed immediately upon arrival in the postanesthesia care unit and were connected to a PCA pump system (Abbott Life Care Infuser, Chicago, IL) containing morphine syringes. The PCA pump was set to deliver a 1.0 - to $1.5-\mathrm{mg}$ intravenous bolus dose of morphine with a lockout time of $5 \mathrm{~min}$, a maximum dose of $40 \mathrm{mg}$ in any 4-h period, and no continuous background infusion. This regimen was overseen by the Acute Pain Service and was continued on the ward for $48 \mathrm{~h}$, during which no other analgesics were administered. For the present analyses, we computed morphine consumption between 24 and $48 \mathrm{~h}$ after surgery.

\section{Data analyses}

Standard descriptive statistics (e.g., frequencies, means, and standard deviations) were calculated for demographic, medical, and psychosocial measures. Chi-squared analyses and $t$ tests were conducted to compare patients with complete longitudinal data (i.e., through 4 weeks after surgery) with patients for whom 4-week postoperative data were missing. Bivariate and multivariate statistical techniques were used to describe the association among preoperative psychological measures of coping and distress and measures of postoperative pain and morphine consumption.
Patterns of bivariate association among the measures were examined using Pearson's Product Moment Correlation Coefficients. Multivariate association was examined using regression analyses. In particular, the multivariate association of postoperative pain with preoperative factors and postoperative NA and morphine consumption was examined by regressing each outcome measure on the explanatory variables. Similarly, we examined the multivariate association of postoperative morphine consumption with preoperative factors, postoperative NA, and pain. To determine the eligible medical or demographic covariates to enter into the equations, we computed the association between the outcome variables and age, anesthesia group assignment, cancer diagnosis (yes/no), pain history (yes/no), and duration of surgery. Age was the only variable significantly associated with any of the outcome variables (morphine consumption after surgery; $r=-.29, P<.001$ ). Therefore, the following variables were included in the model: age, concurrent morphine consumption if the outcome measure was pain or concurrent pain if the outcome measure was morphine consumption, concurrent NA, preoperative distress (MHI, IES, NA), and preoperative coping scale scores. We also ran the same regression models using forward selection for the coping variables on the second step $(P<.05)$ with the other variables forced into the model on the first step. Standard ordinary least-squares graphical and statistical diagnostic procedures were used for the regression analyses. Using graphical and statistical criteria, including studentized residual, DFBeta, DFFit, Cook, and Mahalanobis values, no heteroscedasticity was identified and no observations were signaled for exclusion from the analyses as multivariate outliers or influential points. Furthermore, tolerance and variance inflation factor values did not indicate problematic levels of multicollinearity among the explanatory variables included in the final regression models. Data are presented as mean \pm S.D., unless otherwise stated.

\section{Results}

\section{Descriptive analyses}

One hundred and twenty-two women provided baseline data and completed at least one of the follow-up measures $48 \mathrm{~h}$ after surgery. For the Pearson correlation analyses examining the association of the preoperative measures with the postoperative pain level and morphine consumption, the number of patients in the analyses ranged from 113 to 122 for the 48-h postoperative time point and from 77 to 83 patients for the 4-week follow-up assessment. The hierarchical regression analyses used only data on patients who had complete outcome data for each of the variables in the equation (48-h PRI-T, $n=107$; 48-h morphine consumption, $n=107$; 4-week follow-up, $n=71$ ). There were no statistically significant differences between the patients who completed all the assessments and the patients who were 
not included in the final regression analyses with respect to demographic or baseline psychosocial characteristics. There was, however, a higher proportion of patients with pain history $\left[\chi^{2}(1)=6.2, P=.01\right]$ and a lower proportion of patients with a cancer diagnosis $\left[\chi^{2}(1)=5.6, P=.02\right]$ among the patients who completed all the assessments. However, neither of these factors was associated with either postoperative levels of pain or morphine consumption.

The mean age of the patients was $45 \pm 9$ years (range $23-$ 73 years). Nineteen percent of the patients were undergoing surgery for cancer, and $43 \%$ had a history of pain. The patients were evenly distributed across the anesthesia groups, with $33 \%$ receiving a preincisional epidural, $34 \%$ a postincisional epidural, and $33 \%$ a sham epidural. The average duration of surgery was $91 \pm 35 \mathrm{~min}$ (range 20-193 min). Table 1 presents the data from the preoperative psychosocial measures, and Table 2 presents summary statistics from the postoperative measures of pain, morphine consumption, and NA.

\section{Correlational analyses}

Preoperative correlations between distress and coping, and postoperative $N A$

All the coping subscales were statistically significantly associated with at least one of the preoperative distress measures $(r=.19$ to $.57, P<.05$ to $<.001)$, except for the humor subscale (Table 3). All the measures of preoperative distress were statistically significantly associated with postoperative NA at both time points $\left(r=.36\right.$ to .71 , all $P_{\mathrm{S}}<.001$; Table 3). A number of the coping subscales were also associated with the postoperative NA scores $(r=.23$ to .67 , $P<.05$ to $<.001$ ).

Table 1

Preoperative psychosocial measures

\begin{tabular}{llcc}
\hline Preoperative measure & Mean & S.D. & Range \\
\hline MHI-18 & 84.9 & 12.7 & $35-108$ \\
EES & 23.3 & 15.4 & $0-70$ \\
NA & 27.7 & 10.9 & $10-55$ \\
COPE & & & \\
Self-distraction & 5.0 & 1.9 & $2-8$ \\
Active & 5.3 & 2.0 & $2-8$ \\
Denial & 2.8 & 2.0 & $2-8$ \\
Substance use & 2.3 & 1.0 & $2-8$ \\
Emotional support & 6.9 & 1.4 & $3-8$ \\
Behavioral disengagement & 2.4 & 1.0 & $2-8$ \\
Venting & 4.1 & 1.7 & $2-8$ \\
Reframing & 5.8 & 1.8 & $2-8$ \\
Planning & 5.1 & 2.0 & $2-8$ \\
Humor & 4.2 & 2.0 & $2-8$ \\
Acceptance & 6.7 & 1.4 & $2-8$ \\
Religion & 4.7 & 2.3 & $2-8$ \\
\hline
\end{tabular}

MHI: Mental Health Inventory - possible range 0-108 (high scores =better mental health).

IES: Impact of Event Scale Total score-possible range 0-75 (high scores $=$ worse distress).

NA: negative affect - possible range $0-108$ (high scores $=$ worse NA).
Table 2

Postoperative outcome measures

\begin{tabular}{|c|c|c|c|c|c|c|}
\hline \multirow{2}{*}{$\begin{array}{l}\text { Postoperative } \\
\text { measures }\end{array}$} & \multicolumn{2}{|c|}{$\begin{array}{l}\text { Forty-eight } \\
\text { hour post } \\
\text { after surgery }\end{array}$} & \multirow{2}{*}{$\frac{}{\text { Range }}$} & \multicolumn{2}{|c|}{$\begin{array}{l}\text { Four weeks } \\
\text { after surgery }\end{array}$} & \multirow[b]{2}{*}{ Range } \\
\hline & Mean & S.D. & & Mean & S.D. & \\
\hline PRI-T & 16.0 & 12.2 & $0=58$ & 14.3 & 11.5 & $0-62$ \\
\hline Negative affect & 23.8 & 10.0 & $11-63$ & 19.5 & 9.3 & $10-64$ \\
\hline $\mathrm{MC}$ (mg) & 34.9 & 25.8 & $0-130$ & & & \\
\hline
\end{tabular}

PRI-T: Pain Rating Index - total from the McGill Pain Questionnaire. $\mathrm{MC}$ : morphine consumption $24-48 \mathrm{~h}$ after surgery.

\section{Preoperative variables and postoperative pain and morphine consumption}

Preoperative IES and NA scores were consistently positively associated with pain scores at both time points ( $r=.22$ to $.41, P<.05$ to $<.001$; see Table 3 ). Several coping subscales were associated with postoperative PRI-T scores at both time points ( $r=.19$ to $.47, P<.05$ to $<.001$; Table 3 ). Particularly noteworthy was the consistency in the correlations between the coping subscales of denial, behavioral disengagement, and the use of religious-based coping with PRI-T scores over time: $48 \mathrm{~h}(r=.22, P \leq .01 ; r=.23, P \leq .01$; $r=.26, P<.01$, respectively) and 4 weeks $(r=.31, P<.01$; $r=.47, P<.001 ; r=.46, P<.001$, respectively) after surgery.

The three preoperative distress scores were consistently associated with morphine consumption after surgery $(r=.20$ to $.32, P<.05$ to $\leq .001$; Table 3 ). Endorsement of active $(r=.21, P<.05)$, emotional support $(r=.32, P<.001)$, planning $(r=.19, P<.05)$, and religious-based $(r=.26, P<.01)$ coping was positively associated with morphine consumption after surgery.

\section{Postoperative NA, postoperative pain, and morphine consumption}

Negative affect $48 \mathrm{~h}$ after surgery was associated with PRI-T scores $48 \mathrm{~h}$ after surgery $(r=.36, P<.001)$. Similarly, negative affect 4 weeks after surgery was associated with PRI-T scores 4 weeks after surgery $(r=.46, P<.001)$. NA after surgery was not associated with postoperative morphine consumption $(r=.14)$. However, postoperative pain levels were positively associated with morphine consumption $(r=.32, P<.001)$.

\section{Multivariate analyses}

The results of the regression analysis are presented by showing the factors that were associated with the outcome variables in the final model (i.e., after controlling for all other factors in the model).

Age $(P=.012)$, concurrent PRI-T scores $(P=.027)$, use of emotional support coping ( $P=.031$ ), and religious-based coping $(P=.036)$ were each independently associated with morphine consumption after surgery, and preoperative NA was marginally associated with morphine consumption after surgery $(P=.086)$. When the coping variables were allowed 


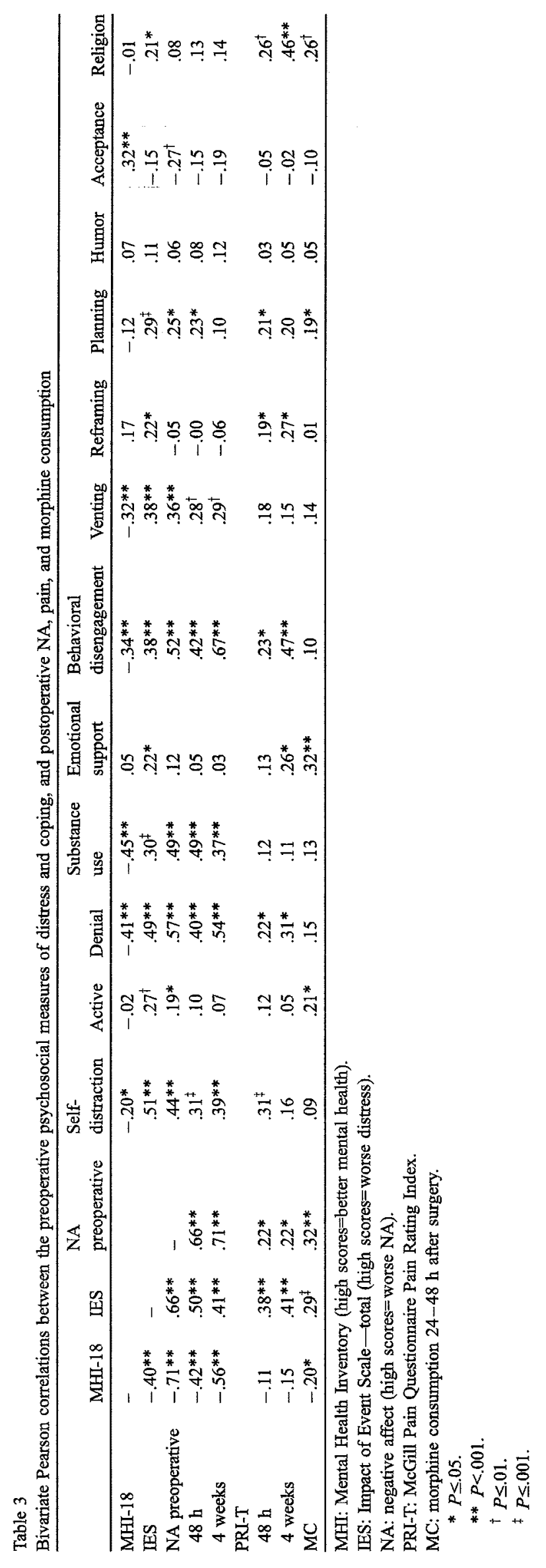


Table 4

Pre- and postoperative variables that were statistically significantly associated with $\mathrm{MC}$ or PRI-T scores from the hierarchical regression models using forced entry

\begin{tabular}{|c|c|c|c|c|c|c|}
\hline \multirow{3}{*}{$\begin{array}{l}\text { Explanatory } \\
\text { variables }\end{array}$} & \multirow{2}{*}{\multicolumn{2}{|c|}{$\begin{array}{l}\mathrm{MC} \\
24-48 \mathrm{~h} \\
\text { Postoperative } \\
\end{array}$}} & \multicolumn{4}{|c|}{ PRI-T } \\
\hline & & & \multicolumn{2}{|c|}{$\begin{array}{l}48 \mathrm{~h} \\
\text { Postoperative }\end{array}$} & \multicolumn{2}{|c|}{$\begin{array}{l}4 \text { Weeks } \\
\text { postoperative }\end{array}$} \\
\hline & $\beta$ & $P$ & $\beta$ & $P$ & $\beta$ & $P$ \\
\hline Age & -.24 & .012 & & & & \\
\hline PRI-T $48 \mathrm{~h}$ & .23 & .027 & & & & \\
\hline $\mathrm{MC}$ & & & .24 & .027 & & \\
\hline NA preoperative & .30 & .086 & -.31 & .078 & -.35 & .041 \\
\hline $\mathrm{NA} 48 \mathrm{~h}$ & & & .25 & .055 & & \\
\hline NA 4 weeks & & & & & .45 & .0009 \\
\hline IES & & & .21 & .10 & .35 & .008 \\
\hline Emotional support & .23 & .031 & & & .21 & .049 \\
\hline $\begin{array}{l}\text { Behavioral } \\
\text { disengagement }\end{array}$ & & & & & .32 & .034 \\
\hline Religion & .21 & .036 & & & .34 & .001 \\
\hline Self-distraction & & & .25 & .039 & & \\
\hline
\end{tabular}

The following variables were included in each model: age; concurrent morphine consumption if the outcome measure was pain or concurrent pain if the outcome measure was morphine consumption; concurrent NA; preoperative distress (MHI, IES, and NA); and preoperative coping scale scores. IES: Impact of Event Scale-total (high scores=worse distress).

NA: negative affect (high scores= worse negative affect).

PRI-T: McGill Pain Questionnaire Pain Rating Index.

$\mathrm{MC}$ : morphine consumption $24-48 \mathrm{hr}$ after surgery.

to enter the model on the second step, using forward selection, the model remained the same (see Table 4).

Few of the preoperative psychosocial variables were associated with PRI-T scores $48 \mathrm{~h}$ after surgery. Morphine consumption after surgery $(P=.027)$ and self-distraction coping $(P=.039)$ were independently positively associated with 48-h PRI-T scores, concurrent NA $(P=.055)$, preoperative NA $(P=.078)$, and preoperative IES scores $(P=.10)$ were marginally associated with PRI-T scores $48 \mathrm{~h}$ after surgery. When the coping variables were allowed to enter the model on the second step, using forward selection, the model remained the same, except that concurrent NA, preoperative NA, and preoperative IES scores were now significantly associated with 48-h PRI-T scores (Table 4).

More of the preoperative psychosocial variables were associated with PRI-T scores 4 weeks after surgery. Preoperative IES scores $(P=.008)$, preoperative NA $(P=.041)$, use of emotional support coping $(P<.049)$, religious-based coping $(P=.001)$, behavioral disengagement coping $(P=.034)$, and concurrent NA $(P=.009)$ were all independently positively associated with PRI-T scores 4 weeks after surgery. When the coping variables were allowed to enter the model on the second step, using forward selection, the model remained the same, except that the use of emotional support coping and behavioral disengagement coping no longer entered the model (Table 4). We also conducted the same regression analyses using the sensory pain rating index (PRI-S) from the MPQ as the measure of pain to remove the influence of the affective adjectives (PRI-A), which could be more strongly associated with preoperative psychosocial factors. The overall findings remained consistent.

\section{Discussion}

One of the most important findings from the present study was the ability of certain preoperative factors to predict postoperative pain levels and analgesic consumption while in the hospital and 4 weeks later, even after accounting for the effects of age and the concurrent factors that were associated with the outcome measures. The results of the present study indicate that preoperative distress and coping predict postoperative morphine consumption and pain level, as measured by the MPQ. The significant associations between preoperative measures of distress and postoperative NA, postoperative NA and pain levels, and postoperative pain and morphine consumption point to the importance of assessing and controlling for concurrent postoperative factors when predicting postoperative recovery. In particular, the findings indicate that the association between preoperative distress and coping and postoperative recovery is independent of a generalized reactive state. This suggests that the association between the preoperative distress and coping strategies and the outcome variables is independent of the more general association found between coping and preoperative distress or postoperative NA.

Few presurgical factors predicted pain and morphine consumption while in the hospital. When preoperative distress was allowed to enter the model prior to the coping variables, preoperative distress was associated with increased pain levels while in the hospital. In addition, in the bivariate analyses, preoperative measures of distress also predicted increased postoperative morphine consumption. The association between preoperative distress and postoperative pain and analgesic consumption is consistent with previous research [1-8]. However, this is one of the few studies that controlled for concurrent postoperative factors when assessing the predictive nature of preoperative psychosocial variables. Importantly, when we controlled for age, concurrent postoperative factors, and preoperative coping, the association between preoperative distress and pain or morphine consumption was no longer significant. This suggests that coping strategies may be more predictive of pain and analgesic consumption while in the hospital than is preoperative distress.

The endorsement of certain coping strategies preoperatively predicted postoperative pain levels and morphine consumption while in the hospital. In the bivariate analyses, the more passive/avoidant coping strategies were most strongly positively associated with pain and morphine consumption. In the multivariate analyses, preoperative endorsement of coping by self-distraction predicted increased pain levels while in the hospital. In addition, reported use of either emotional support or religious-based coping independently predicted increased use of analgesic 
medication in the hospital, even after accounting for concurrent NA and pain levels. Therefore, regardless of the patients' level of pain and NA while in the hospital, the more they reported engaging in these passive forms of coping before surgery, the more morphine they selfadministered while in the hospital.

Presurgical distress and endorsement of certain coping strategies were more consistently associated with pain 4 weeks after surgery. For example, in the multivariate analyses, preoperative measures of distress predicted increased pain levels 4 weeks after surgery. This indicates that the higher the level of preoperative distress, the higher the level of pain 4 weeks after surgery, even after accounting for the effects of age, the preoperative coping strategies, and NA at the time of assessment. In addition, preoperative endorsement of emotional support coping, behavioral disengagement, and religious-based coping predicted increased pain levels 4 weeks after surgery after accounting for age, preoperative levels of distress, the other coping subscales, and concurrent NA.

Overall, the most consistent finding in the multivariate analyses was the association between religious-based and emotional support coping with both postoperative pain and morphine consumption. Although religious-based coping is sometimes viewed as an active coping strategy [27], the measure used in the present study involves engaging in passive coping efforts ("I've been trying to find comfort in my religion or spiritual beliefs...I've been praying or meditating"). The association between preoperative coping and postoperative outcomes is consistent with the results of other studies $[3,8]$. However, this is one of the only studies that effectively controlled for concurrent factors that could influence pain and analgesic consumption and the association between preoperative distress and coping.

It is not clear why engaging in these passive forms of coping and relying on supportive others is associated with increased pain reporting and use of analgesic medications. It may be that patients who use these forms of coping are more willing to express their level of pain and are encouraged to use analgesic medication. Alternatively, preoperative distress and use of passive forms of coping may predispose patients to be more attentive to their current pain state [28]. It should be noted, however, that the coping scale in the present study was completed preoperatively and addressed the question of the coping strategies that patients used before surgery and not in the days and weeks after surgery while in pain. Some research suggests that, although coping strategies will be situation specific, how one responds in a given situation may be a relatively stable individual characteristic [29]. Therefore, it may not necessarily be the use of religious-based, emotional support, and other forms of passive coping to directly manage pain per se that is associated with worse outcomes, but patients who report engaging in these forms of coping to cope with an upcoming surgery end up reporting more pain and using more analgesic medications after surgery.
There are a number of factors that may limit the generalizability of the current findings. For example, we cannot exclude the possibility that presurgical pain levels may have influenced the outcomes of this study because we did not assess pain intensity before surgery. However, in the analyses, we did examine the influence of pain history. Importantly, although this was a longitudinal, prospective study, it is correlational in nature and, therefore, it is not possible to infer causality between the preoperative psychosocial measures and postoperative pain levels and morphine consumption. Some other factor or factors may be responsible for the association between variables. In addition, because the MPQ is a multidimensional measure of pain, it appropriately incorporates the affective aspects of the pain experience. Therefore, it could be argued that the use of this measure might increase the probability of finding an association between preoperative psychosocial factors and postoperative pain by virtue of the affective overlap between these sets of variables and not because the former variables are intrinsically related to pain. However, the results remained consistent even when only the PRI-S of the MPQ was examined. Finally, the loss of patients to follow-up by the 4-week assessment may have introduced a bias into the study. It is possible, for example, that patients experiencing more pain and those using ineffective coping may have been overrepresented at the 4-week follow-up. It should be noted, however, that we did not find significant differences in the preoperative psychosocial variables between patients who were contacted and those who were lost to the 4-week follow-up.

The ability to predict postoperative pain and analgesic consumption based on preoperative psychosocial factors suggests that patients can be screened preoperatively in an effort to enhance postoperative recovery. In addition, consistent with the results of Kain et al. [8], the more reactive components of distress before surgery, such as NA, were better predictors of pain than were more stable measures of mental health such as depression or trait anxiety (e.g., the MHI). This raises the possibility that presurgical programs can be developed to decrease levels of distress and help patients engage in adaptive coping strategies prior to undergoing major surgery. Although there is some evidence that such intervention programs would be useful adjuncts to standard care [30], their efficacy remain equivocal [31] and difficult to interpret due to small sample sizes, heterogeneous patient populations, and the use of nonvalidated outcome measures. Large-scale studies are needed that examine the potential benefits of such a program on postoperative adjustment and the development of long-term pain.

Recent efforts to identify factors associated with the transition of acute postoperative pain to chronic postsurgical pain have focused primarily on the role of biomedical variables in the acute postoperative phase [32]. For example, two of the most robust findings are that acute postoperative pain [33] and/or consumption of analgesics [34-36] predict the development of long-term pain $[32,37]$. In contrast, very little research has focused on the preoperative psychosocial 
predictors of long-term pain. Some research suggests that certain personality factors (e.g., neuroticism or being introverted) predict long-term pain and symptoms after surgery [38-40]. These factors, however, are relatively stable and difficult to modify. The results of the present study show that patients who reported coping with the stress of their upcoming surgery by making use of emotional support, behavioral disengagement, or religious-based strategies had increased pain levels 4 weeks after surgery (even after accounting for age, preoperative levels of distress, the other coping subscales, and concurrent NA). We do not know what factor or factors are responsible for the increased pain in these patients and how the use of these coping strategies is involved. We also do not know whether this result would be maintained at a longer follow-up. However, the results suggest that a fruitful approach to the study of the transition of acute pain to long-term pain could involve the assessment of coping strategies prior to surgery.

\section{Acknowledgments}

Supported by Grants MT-12052 and MOP-37845 from the Canadian Institutes of Health Research (CIHR), Ontario, Canada; Grant NS35480 from the National Institute of Neurolgical Disorders and Stroke, Bethesda, MD; and a CIHR Investigator Award to Dr. Katz. We thank Beth Notzon, from the Department of Scientific Publications, The University of Texas M.D. Anderson Cancer Center, for her helpful editorial comments on this article.

\section{References}

[1] de Groot KI, Boeke S, van den Berge HJ, Duivenvoorden HJ, Bonke $B$, Passchier J. The influence of psychological variables on postoperative anxiety and physical complaints in patients undergoing lumbar surgery. Pain 1997;69(1-2):19-25.

[2] Johnson JE, Leventhal $H$, Dabbs JM. Contribution of emotional and instrumental response processes in adaptation to surgery. J Pers Soc Psychol 1971;20(1):55-64.

[3] Mathews A, Ridgeway V. Personality and surgical recovery: a review. Br J Clin Psychol 1981;20(Pt 4):243-60.

[4] Scott LE, Clum GA, Peoples IB. Preoperative predictors of postoperative pain. Pain 1983;15(3):283-93.

[5] Taenzer P, Melzack R, Jeans ME. Influence of psychological factors on postoperative pain, mood and analgesic requirements. Pain 1986;24(3):331-42.

[6] Feinmann C, Ong M, Harvey W, Harris M. Psychological factors influencing post-operative pain and analgesic consumption. Br J Oral Maxillofac Surg 1987;25(4):285-92.

[7] Perry F, Parker RK, White PF, Clifford PA. Role of psychological factors in postoperative pain control and recovery with patientcontrolled analgesia. Clin J Pain 1994;10(1):57-63 [discussion 82-5].

[8] Kain ZN, Sevarino F, Alexander GM, Pincus S, Mayes LC. Preoperative anxiety and postoperative pain in women undergoing hysterectomy. A repeated-measures design. J Psychosom Res 2000;49(6):417-22.

[9] Wise TN, Hall WA, Wong $O$. The relationship of cognitive styles and affective status to post-operative analgesic utilization. J Psychosom Res 1978;22(6):513-8.
[10] Johnston M, Carpenter L. Relationship between pre-operative anxiety and post-operative state. Psychol Med 1980;10(2):361 - 7.

[11] Ray C, Fitzgibbon G. Stress arousal and coping with surgery. Psychol Med 1981;11(4):741-6.

[12] Salmon P, Evans R, Humphrey DE. Anxiety and endocrine changes in surgical patients. Br J Clin Psychol 1986;25(Pt 2):135-41.

[13] Wallace LM. Pre-operative state anxiety as a mediator of psychological adjustment to and recovery from surgery. $\mathrm{Br} \mathrm{J}$ Med Psychol 1986;59(Pt 3):253-61

[14] Voulgari A, Lykouras L, Papanikolaou M, Tzonou A, DanouRoussaki A, Christodoulou G. Influence of psychological and clinical factors on postoperative pain and narcotic consumption. Psychother Psychosom 1991;55(2-4):191-6.

[15] Boeke S, Duivenvoorden HJ, Verhage F, Zwaveling A. Prediction of postoperative pain and duration of hospitalization using two anxiety measures. Pain 1991;45(3):293--7.

[16] Lazarus RS, Folkman S. Stress, appraisal, and coping. New York: Springer, 1984.

[17] George JM, Scott DS, Turner SP, Gregg JM. The effects of psychological factors and physical trauma on recovery from oral surgery. J Behav Med 1980;3(3):291-310.

[18] Carver CS, Scheier MF, Weintraub JK. Assessing coping strategies: a theoretically based approach. J Pers Soc Psychol 1989;56:267-83.

[19] Carver CS. You want to measure coping but your protocol's too long: consider the brief COPE. Int J Behav Med 1997;4:92-100.

[20] Ware JE, Johnston SA, Davies-Avery A, Brook RH. Conceptualization and measurement of health for adults in the Health Insurance Study (Mental Health R-1987/3-HEW: 3). Santa Monica (CA): RAND, 1994.

[21] Weinstein MC, Berwick DM, Goldman PA, Murphy JM, Barsky AJ. A comparison of three psychiatric screening tests using receiver operating characteristic (ROC) analysis. Med Care 1989;27(6): 593-607.

[22] Horowitz M, Wilner N, Alvarez W. Impact of Events Scale: measure of subjective stress. Psychosom Med 1979;41:209-18.

[23] Zakowski SG, McAllister CG, Deal M, Baum A. Stress, reactivity, and immune function in healthy men. Health Psychol 1992;11(4):223-32.

[24] Zakowski SG, Cohen L, Hall MH, Wollman K, Baum A. Differential effects of active and passive laboratory stressors on immune function in a group of healthy men. Int J Behav Med 1994;1(1):163-84

[25] Melzack R. The McGill Pain Questionnaire: major properties and scoring methods. Pain 1975;1:277-99.

[26] Katz J, Melzack R. Measurement of pain. Surg Clin North Am 1999;79:231-52.

[27] Koenig HG, Cohen HJ, George LK, Hays JC, Larson DB, Blazer DG, Attendance at religious services, interleukin- 6 , and other biological parameters of immune function in older adults. Int J Psychiatry Med 1997;27(3):233-50.

[28] Nelson FV, Zimmerman L, Barnason S, Nieveen J, Schmaderer M. The relationship and influence of anxiety on postoperative pain in the coronary artery bypass graft patient. J Pain Symptom Manage 1998;15(2):102-9.

[29] Lazarus RS. Coping theory and research: past, present, and future. Psychosom Med 1993;55(3):234-47.

[30] Ludwick-Rosenthal $R$, Neufeld WJ. Stress management during noxious medical procedures: an evaluation review of outcome studies. Psychol Bull 1988;104:326- 42.

[31] Johnston $M$, Vogele $C$. Benefits of psychological preparation for surgery: a meta-analysis. Ann Behav Med 1993;15:245-56.

[32] Perkins FM, Kehlet $H$. Chronic pain as an outcome of surgery. A review of predictive factors. Anesthesiology 2000;93(4):1123-33.

[33] Katz J, Jackson M, Kavanagh BP, Sandler AN. Acute pain after thoracic surgery predicts long-term post-thoracotomy pain. Clin J Pain $1996 ; 12: 50-5$.

[34] Haythornthwaite JA, Raja SN, Fisher B, Frank SM, Brendler CB, Shir Y. Pain and quality of life following radical retropubic prostatectomy. J Urol 1998;160(5):1761-4.

[35] Perttunen K, Tasmuth T, Kalso E. Chronic pain after thoracic surgery: a follow-up study. Acta Anaesthesiol Scand 1999;43(5):563-7. 